\title{
Structural, Spectral, Thermodynamic and HOMO, LUMO Analysis of 2, 6 dithenobenzene-3-enyl 3,5 dimethyl piperdine-4-one: A quantum chemical analysis
}

\author{
SANGEETHA. R. $\mathrm{K}^{1}$ and AYYAPPAN. $\mathbf{S}^{2 *}$ \\ 'Department of physics, Sri Eshwar College of Engineering, Coimbatore, India. \\ 2Department of physics, Government College of Technology, Coimbatore, India. \\ ${ }^{*}$ Corresponding author E-mail: ayyappan@gct.ac.in \\ http://dx.doi.org/10.13005/ojc/370618
}

(Received: September 27, 2021; Accepted: November 05, 2021)

\begin{abstract}
In the current work, the vibrational frequencies, infrared intensities, molecular geometry and Raman scattering were determined and investigated using ab initio Hartree-Fock (HF) and density functional methods with a basis set of $6-311++G(d, p)$ of the organic molecule under interpretation. The FT-IR and FT-Raman spectra of titled molecule have been recorded in the region 4000-400 $\mathrm{cm}^{-1}$ and $5000-70 \mathrm{~cm}^{-1}$, respectively. The optimized geometry structures (bond lengths and bond angles) achieved using HF shows the best result with the experimental values of the titled molecule. The frontier molecular orbitals help to distinguish chemical responsiveness and molecular kinetic steadiness, thus HOMO-LUMO analysis can be done using the quantum chemistry to improve thermodynamics. The electron density mapping to electrostatic potential surfaces were involved in finding the reactivity sites of the titled compound. With the help of Gauss view 5.0 and Chemcraft packages, the obtained outputs are analyzed. Hyperpolarizability and non-linear optical effect of isolated molecules of NLO materials are observed to be an extensive tool for molecular spectroscopy research. Therefore, for industrial application, Hyperpolarizability of the molecule is also studied.
\end{abstract}

Keywords: DFT, HF, NBO, HOMO LUMO, NLO.

\section{INTRODUCTION}

The phenomenal developments in ab initio and density functional theory (DFT) over the last decade have made possible efficient and systematic predictions of harmonic vibrational frequencies, together with harmonic dipole strengths and rotational strengths which determine harmonic intensities of fundamental transitions in vibrational non polarized absorption like infra-red (IR) and vibrational circular dichroism (VCD) spectra. Molecular computational studies can provide a broad understanding of the relationships between molecular architecture, non-linear optical characteristics and bioactivity ${ }^{1}$. Piperidins-4-one and its derivatives are highly determined due to its pharmaceutical applications, including antiviral drugs, anti-tumor drugs ${ }^{2}$, analgesics and local anesthetic ${ }^{3,4}$, bactericides, fungicides, and depressants. It is very sensitive to spectroscopic investigation over the past few years ${ }^{5,6}$. The current

This is an Open Access article licensed under a Creative Commons license: Attribution 4.0 International (CC- BY). Published by Oriental Scientific Publishing Company @ 2018

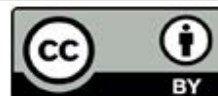


study aimed to give a complete outline of the optimized molecule structure, the vibrational assignments, electronic characteristics like an energy gap, a HOMO, a LUMO and an electrostatic molecular potential of the titled compound. In order to calculate the number of vibrational waves, the optimized molecular structure was used in B3LYP method. This paper focuses on the spectral characterization of the title molecules ${ }^{7-8}$. The correlation between the title molecular structure, bioactivity and Non-Linear optical characters are also decoded with quantum chemical calculations. A systematic approach presents unique applications of quantum mechanical method in the analysis of sum biological important piperidine derivatives compounds.

\section{Computational details}

The compound was optimized with 6-31G $(d, p)$ basis set using DFT/B3LYP quantum chemical methods. For the calculation of frontier molecular orbitals, a time-dependent DFT (TD-DFT) method was used $^{9-11}$. DFT (B3LYP/6-311++G (d, p) method has been employed in this work to calculate optimized molecular structure, vibrational descriptions and charge transfer of the titled molecule. DFT calculations were significantly employed in electronic molecular structure treatment. The investigation was done under $6311++G$ is the basis $(d, p)$ because of an efficient and cost effective level ${ }^{12}$. The complete computation has been carried out on personal computers involved with the Gaussian 09W programme package.

\section{RESULTS AND DISCUSSION}

\section{Molecular geometry}

Gaussian 09 and Gauss view programme provides all the optimized structure and numbering of atoms in the selected molecule. The bond length and bond angles of the optimized molecule are calculated with the basis set of HF/B3LYP using 6-31G. The calculated B3LYP values are higher than HF levels. The bond length and angles are presented in Table 1. Considering the energy-optimized geometry of the molecular derived from both basis set, the conceptual values of this method for correlation were taken up and are dependable. The $\mathrm{C}-\mathrm{C}$ and $\mathrm{C}-\mathrm{H}$ bond distances of piperidine ring were in the range of 1.555-1.516 $\AA$ and 1.112-1.094 $\AA$ respectively, whereas, the optimized $\mathrm{C}-\mathrm{C}-\mathrm{C}$ and $\mathrm{C}-\mathrm{C}-\mathrm{H}$ bond angles were in the range of $113.60-109.99^{\circ}$ and $111.12-103.57^{\circ}$ respectively. The $\mathrm{C}-\mathrm{N}$ and $\mathrm{N}-\mathrm{H}$ bond distances were calculated in the range 1.09-1.07 $\AA$ and $1.01 \AA, 0.9 \AA$, also calculated the $\left(126.6^{\circ}\right)$ $\mathrm{C}-\mathrm{C}-\mathrm{C},\left(108.5^{\circ}\right) \mathrm{N}-\mathrm{C}-\mathrm{C}$ bond angles for the piperidine molecule. With regard to the Endo cyclic torsional angles, the optimized $\mathrm{C}-\mathrm{H}$ lengths in the $\mathrm{CH}_{2}$ groups are calculated using the B3LYP method in the $1.092 \AA$ A range. In addition, calculation outcome shows that the geometric parameter calculated is in good understanding with the remaining geometric parameters.

Table 1: Optimized some geometrical parameters of 2,6 dithenobenzene-3-enyl 3,5 dimethyl piperidine-4-one bond length $(\mathrm{A})$, bond angles $\left(^{\circ}\right)$ and dihedral angle

Optimized Parameters (Angstroms and degrees)

Atom Bonds

$\begin{array}{ll}\text { Value } & \\ \text { HF/6-31G } & \text { B3LYP/6-31G }\end{array}$

\begin{tabular}{|c|c|c|}
\hline \multicolumn{3}{|c|}{ Bond Lengths } \\
\hline $\mathrm{C} 1-\mathrm{C} 2$ & 1.5417 & 1.549 \\
\hline C1-C3 & 1.5251 & 1.5497 \\
\hline $\mathrm{C} 1-\mathrm{C} 26$ & 1.5373 & 1.5495 \\
\hline $\mathrm{C} 1-\mathrm{H} 28$ & 1.0828 & 1.0962 \\
\hline $\mathrm{C} 2-\mathrm{N} 4$ & 1.4588 & 1.476 \\
\hline $\mathrm{C} 2-\mathrm{C} 7$ & 1.5524 & 1.5645 \\
\hline $\mathrm{C} 2-\mathrm{H} 29$ & 1.0924 & 1.1072 \\
\hline C3-C5 & 1.5251 & 1.5296 \\
\hline C3-O27 & 1.1952 & 1.2445 \\
\hline N4-C6 & 1.4588 & 1.476 \\
\hline $\mathrm{N} 4-\mathrm{H} 30$ & 0.997 & 1.0156 \\
\hline C5-C6 & 1.5416 & 1.5495 \\
\hline C5-C25 & 1.5373 & 1.5496 \\
\hline $\mathrm{C} 5-\mathrm{H} 31$ & 1.0828 & 1.0962 \\
\hline C6-C16 & 1.5525 & 1.5645 \\
\hline C6-H32 & 1.0924 & 1.1072 \\
\hline C7-C8 & 1.5208 & 1.5243 \\
\hline C7-C9 & 1.5452 & 1.5491 \\
\hline C7-H33 & 1.0862 & 1.0988 \\
\hline $\mathrm{C} 8-\mathrm{C} 10$ & 1.3913 & 1.4065 \\
\hline $\mathrm{C} 8-\mathrm{C} 12$ & 1.3849 & 1.4001 \\
\hline C9-S11 & 1.826 & 1.9153 \\
\hline $\mathrm{C} 9-\mathrm{H} 34$ & 1.0826 & 1.092 \\
\hline C9-H35 & 1.0814 & 1.091 \\
\hline C10-S11 & 1.7736 & 1.8414 \\
\hline C10-C14 & 1.3826 & 1.3937 \\
\hline C12-C13 & 1.3876 & 1.4022 \\
\hline $\mathrm{C} 12-\mathrm{H} 36$ & 1.0756 & 1.0854 \\
\hline C13-C15 & 1.3851 & 1.3998 \\
\hline $\mathrm{C} 13-\mathrm{H} 37$ & 1.0752 & 1.0848 \\
\hline C14-C15 & 1.3868 & 1.4023 \\
\hline $\mathrm{C} 14-\mathrm{H} 38$ & 1.0751 & 1.0844 \\
\hline $\mathrm{C} 15-\mathrm{H} 39$ & 1.0756 & 1.0852 \\
\hline $\mathrm{C} 16-\mathrm{C} 17$ & 1.5452 & 1.5491 \\
\hline C16-C18 & 1.5207 & 1.5243 \\
\hline $\mathrm{C} 16-\mathrm{H} 40$ & 1.0862 & 1.0988 \\
\hline C17-S19 & 1.826 & 1.9154 \\
\hline
\end{tabular}




\begin{tabular}{|c|c|c|c|c|c|}
\hline $\mathrm{C} 17-\mathrm{H} 41$ & 1.0814 & 1.091 & Continue Tabel 1 & & \\
\hline $\mathrm{C} 17-\mathrm{H} 42$ & 1.0826 & 1.092 & C7-C9-H34 & 110.5167 & 111.741 \\
\hline C18-C20 & 1.3913 & 1.4065 & C7-C9-H35 & 113.9293 & 114.0752 \\
\hline C18-C21 & 1.3849 & 1.4001 & S11-C9-H34 & 108.4989 & 107.264 \\
\hline $\mathrm{S} 19-\mathrm{C} 20$ & 1.7736 & 1.8414 & S11-C9-H35 & 108.4278 & 107.2119 \\
\hline C20-C23 & 1.3826 & 1.3937 & $\mathrm{H} 34-\mathrm{C} 9-\mathrm{H} 35$ & 108.4586 & 109.6694 \\
\hline C21-C22 & 1.3876 & 1.4023 & C8-C10-S11 & 112.7779 & 112.8978 \\
\hline $\mathrm{C} 21-\mathrm{H} 43$ & 1.0756 & 1.0854 & C8-C10-C14 & 121.492 & 121.8204 \\
\hline C22-C24 & 1.3851 & 1.3997 & S11-C10-C14 & 125.7023 & 125.2487 \\
\hline $\mathrm{C} 22-\mathrm{H} 44$ & 1.0752 & 1.0848 & C9-S11-C10 & 90.0302 & 88.3309 \\
\hline C23-C24 & 1.3867 & 1.4024 & $\mathrm{C} 8-\mathrm{C} 12-\mathrm{C} 13$ & 120.0829 & 120.1154 \\
\hline C23-H45 & 1.075 & 1.0844 & $\mathrm{C} 8-\mathrm{C} 12-\mathrm{H} 36$ & 120.3126 & 120.1069 \\
\hline $\mathrm{C} 24-\mathrm{H} 46$ & 1.076 & 1.0852 & С13-C12-H36 & 119.6043 & 119.777 \\
\hline C25-H47 & 1.0833 & 1.094 & C12-C13-C15 & 120.1718 & 120.2002 \\
\hline $\mathrm{C} 25-\mathrm{H} 48$ & 1.0823 & 1.0936 & C12-C13-H37 & 119.7916 & 119.7407 \\
\hline $\mathrm{C} 25-\mathrm{H} 49$ & 1.0862 & 1.0966 & C15-C13-H37 & 120.0358 & 120.0579 \\
\hline $\mathrm{C} 26-\mathrm{H} 50$ & 1.0833 & 1.094 & C10-C14-C15 & 118.8108 & 118.6902 \\
\hline $\mathrm{C} 26-\mathrm{H} 51$ & 1.0862 & 1.0966 & C10-C14-H38 & 120.6631 & 120.7737 \\
\hline C26-H52 & 1.0823 & 1.0936 & C15-C14-H38 & 120.5133 & 120.5219 \\
\hline Bond Angles & & & C13-C15-C14 & 120.419 & 120.3971 \\
\hline $\mathrm{C} 2-\mathrm{C} 1-\mathrm{C} 3$ & 112.6602 & 112.9138 & С13-С15-Н39 & 119.9713 & 120.0557 \\
\hline C2-C1-C2 & 113.4293 & 112.9987 & C14-C15-H39 & 119.5938 & 119.533 \\
\hline $\mathrm{C} 2-\mathrm{C} 1-\mathrm{H} 28$ & 109.5428 & 109.2076 & C6-C16-C17 & 111.0768 & 110.8205 \\
\hline C3-C1-C26 & 108.8228 & 109.1232 & $\mathrm{C} 6-\mathrm{C} 16-\mathrm{C} 18$ & 115.6384 & 114.8327 \\
\hline $\mathrm{C} 3-\mathrm{C} 1-\mathrm{H} 28$ & 103.5984 & 104.3159 & $\mathrm{C} 6-\mathrm{C} 16-\mathrm{H} 40$ & 106.6519 & 106.0655 \\
\hline C26-C1-C28 & 108.2511 & 107.8063 & C17-C16-C18 & 103.8824 & 105.4939 \\
\hline $\mathrm{C} 1-\mathrm{C} 2-\mathrm{N} 4$ & 107.6346 & 107.8015 & $\mathrm{C} 17-\mathrm{C} 16-\mathrm{H} 40$ & 109.4246 & 109.3534 \\
\hline $\mathrm{C} 1-\mathrm{C} 2-\mathrm{C} 7$ & 114.4285 & 114.2842 & $\mathrm{~S} 11-\mathrm{C} 6-\mathrm{H} 40$ & 110.1082 & 110.25 \\
\hline $\mathrm{C}_{1-\mathrm{C} 2-\mathrm{H} 29}$ & 108.5329 & 108.5148 & C16-C17-S19 & 106.8607 & 106.504 \\
\hline $\mathrm{N} 4-\mathrm{C} 2-\mathrm{C} 7$ & 107.8985 & 108.0588 & $\mathrm{C} 16-\mathrm{C} 17-\mathrm{H} 41$ & 113.9237 & 114.0758 \\
\hline N4-C2-H29 & 110.6928 & 110.8025 & $\mathrm{C} 16-\mathrm{C} 17-\mathrm{H} 42$ & 110.5226 & 111.7475 \\
\hline C7-C2-H29 & 107.6648 & 107.3984 & $\mathrm{~S} 19-\mathrm{C} 17-\mathrm{H} 41$ & 108.4244 & 107.2059 \\
\hline C1-C3-C5 & 121.4356 & 121.7206 & $\mathrm{~S} 19-\mathrm{C} 17-\mathrm{H} 42$ & 108.5014 & 107.2615 \\
\hline C1-C3-O27 & 119.2528 & 119.0697 & $\mathrm{H} 41-\mathrm{C} 17-\mathrm{H} 42$ & 108.4605 & 109.6715 \\
\hline C5-C3-O27 & 119.2543 & 119.0698 & C16-C18-C20 & 114.1291 & 114.8709 \\
\hline C2-N4-C6 & 113.3893 & 114.0966 & $\mathrm{C} 16-\mathrm{C} 18-\mathrm{C} 21$ & 126.912 & 126.4244 \\
\hline $\mathrm{C} 2-\mathrm{N} 4-\mathrm{H} 30$ & 110.7439 & 113.1252 & $\mathrm{C} 20-\mathrm{C} 18-\mathrm{C} 21$ & 118.9463 & 118.7039 \\
\hline C6-N4-H30 & 110.7426 & 113.1234 & C17-S19-C20 & 90.0328 & 88.3325 \\
\hline C3-C5-C6 & 112.6526 & 112.9207 & C18-C20-S19 & 112.7783 & 112.90 \\
\hline C3-C5-C25 & 108.8214 & 109.1214 & $\mathrm{C} 18-\mathrm{C} 20-\mathrm{C} 23$ & 121.4924 & 121.82 \\
\hline С3-C5-H31 & 103.6013 & 104.3184 & S19-C20-C23 & 125.7017 & 125.24 \\
\hline C6-C5-C25 & 113.4331 & 112.9869 & $\mathrm{C} 18-\mathrm{C} 21-\mathrm{C} 22$ & 120.0826 & 120.116 \\
\hline C6-C5-H31 & 109.5412 & 109.2149 & $\mathrm{C} 18-\mathrm{C} 21-\mathrm{H} 43$ & 120.3129 & 120.109 \\
\hline $\mathrm{C} 25-\mathrm{C} 5-\mathrm{H} 31$ & 108.2554 & 107.804 & $\mathrm{C} 22-\mathrm{C} 21-\mathrm{H} 43$ & 119.6042 & 119.773 \\
\hline N4-C6-C5 & 107.6327 & 107.8064 & C21-C22-C24 & 120.172 & 120.200 \\
\hline N4-C6-C16 & 107.8921 & 108.0583 & $\mathrm{C} 21-\mathrm{C} 22-\mathrm{H} 44$ & 119.792 & 119.738 \\
\hline N4-C6-H32 & 110.6928 & 110.8017 & C24-C22-H44 & 120.035 & 120.059 \\
\hline C5-C6-C16 & 114.4384 & 114.2844 & C20-C23-H24 & 118.810 & 118.690 \\
\hline $\mathrm{C} 5-\mathrm{C} 6-\mathrm{H} 32$ & 108.5313 & 108.5122 & C2O-C23-H45 & 120.662 & 120.773 \\
\hline $\mathrm{C} 16-\mathrm{C} 6-\mathrm{H} 32$ & 107.6647 & 107.3971 & C24-C23-H45 & 120.510 & 120.520 \\
\hline C2-C7-C8 & 115.6256 & 114.8313 & C22-C24-C23 & 120.419 & 120.39 \\
\hline $\mathrm{C} 2-\mathrm{C} 7-\mathrm{C} 9$ & 111.0861 & 110.8269 & $\mathrm{C} 22-\mathrm{C} 24-\mathrm{H} 46$ & 119.9703 & 120.05 \\
\hline $\mathrm{C} 2-\mathrm{C} 7-\mathrm{H} 33$ & 106.6554 & 106.0653 & C23-C24-C6 & 119.5948 & 119.533 \\
\hline C8-C7-C9 & 103.8774 & 105.4896 & $\mathrm{C} 5-\mathrm{C} 25-\mathrm{H} 47$ & 110.0854 & 110.034 \\
\hline C8-C7-H33 & 110.1134 & 110.2515 & $\mathrm{C} 5-\mathrm{C} 25-\mathrm{H} 48$ & 110.9354 & 110.593 \\
\hline $\mathrm{C} 9-\mathrm{C} 7-\mathrm{H} 33$ & 109.4246 & 109.3515 & $\mathrm{C} 5-\mathrm{C} 25-\mathrm{H} 49$ & 111.232 & 111.107 \\
\hline C7-C8-C10 & 114.1275 & 114.8713 & $\mathrm{H} 47-\mathrm{C} 25-\mathrm{H} 48$ & 108.4952 & 108.594 \\
\hline C7-C8-C12 & 126.9138 & 126.4214 & $\mathrm{H} 47-\mathrm{C} 25-\mathrm{H} 49$ & 108.3015 & 108.491 \\
\hline C10-C8-C12 & 118.9461 & 118.7066 & $\mathrm{H} 48-\mathrm{C} 25-\mathrm{H} 49$ & 107.6915 & 107.939 \\
\hline C7-C9-S11 & 106.8619 & 106.5058 & $\mathrm{C} 1-\mathrm{C} 26-\mathrm{H} 50$ & 110.0866 & 110.030 \\
\hline \multicolumn{3}{|c|}{ Continue Tabel 1} & $\mathrm{C} 1-\mathrm{C} 26-\mathrm{H} 51$ & 111.2336 & 111.107 \\
\hline
\end{tabular}









\begin{tabular}{|c|c|c|}
\hline $\mathrm{C} 8-\mathrm{C} 12-\mathrm{C} 13-\mathrm{H} 37$ & -179.83 & -179 \\
\hline H36-C12-C13-C15 & -179.32 & -179 \\
\hline $\mathrm{H} 36-\mathrm{C} 12-\mathrm{C} 13-\mathrm{H} 37$ & 0.33 & 0.32 \\
\hline C12-C13-C15-C14 & 1.41 & 1.35 \\
\hline $\mathrm{C} 12-\mathrm{C} 13-\mathrm{C} 15-\mathrm{H} 39$ & 179.9 & 179 \\
\hline H37-C13-C15-C14 & -178.23 & -178 \\
\hline H37-C13-C15-H39 & 0.30 & 0.3 \\
\hline C10-C14-C15-C13 & -1.05 & -0.93 \\
\hline $\mathrm{C} 10-\mathrm{C} 14-\mathrm{C} 15-\mathrm{H} 39$ & -179.60 & -179.5 \\
\hline $\mathrm{H} 38-\mathrm{C} 14-\mathrm{C} 15-\mathrm{C} 13$ & 177.64 & 177.70 \\
\hline $\mathrm{H} 38-\mathrm{C} 14-\mathrm{C} 15-\mathrm{H} 39$ & -0.90 & -0.93 \\
\hline C6-C16-C17-S19 & 89.31 & 89.4 \\
\hline C6-C16-C17-H41 & -30.40 & -28.58 \\
\hline C6-C16-C17-H42 & -152.81 & -153.7 \\
\hline C18-C16-C17-S19 & -35.64 & -35.40 \\
\hline $\mathrm{C} 18-\mathrm{C} 16-\mathrm{C} 17-\mathrm{H} 41$ & -155.36 & -153.45 \\
\hline $\mathrm{C} 18-\mathrm{C} 16-\mathrm{C} 17-\mathrm{H} 42$ & 82.23 & 81.43 \\
\hline $\mathrm{H} 40-\mathrm{C} 16-\mathrm{C} 17-\mathrm{S} 19$ & -153.20 & -153.95 \\
\hline $\mathrm{H} 40-\mathrm{C} 16-\mathrm{C} 17-\mathrm{H} 41$ & 87.08 & 87.98 \\
\hline $\mathrm{H} 40-\mathrm{C} 16-\mathrm{C} 17-\mathrm{H} 42$ & -35.32 & -37.12 \\
\hline C6-C16-C18-C20 & -96.22 & -95.55 \\
\hline $\mathrm{C} 6-\mathrm{C} 16-\mathrm{C} 18-\mathrm{C} 21$ & 85.08 & 84.76 \\
\hline C17-C16-C18-C20 & 25.74 & 26.76 \\
\hline $\mathrm{C} 17-\mathrm{C} 16-\mathrm{C} 18-\mathrm{C} 21$ & -152.93 & -152.91 \\
\hline $\mathrm{H} 40-\mathrm{C} 16-\mathrm{C} 18-\mathrm{C} 20$ & 142.83 & 144.72 \\
\hline $\mathrm{H} 40-\mathrm{C} 16-\mathrm{C} 18-\mathrm{C} 21$ & -35.85 & -34.95 \\
\hline C16-C17-S19-C20 & 30.25 & 28.88 \\
\hline H41-C17-S19-C20 & 153.45 & 151.37 \\
\hline H42-C17-S19-C20 & -88.93 & -90.90 \\
\hline C16-C18-C20-S19 & -3.70 & -4.69 \\
\hline C16-C18-C20-C23 & 178.09 & 177.29 \\
\hline C21-C18-C20-S19 & 175.09 & 175.01 \\
\hline $\mathrm{C} 21-\mathrm{C} 18-\mathrm{C} 20-\mathrm{C} 23$ & -3.10 & -2.99 \\
\hline $\mathrm{C} 16-\mathrm{C} 18-\mathrm{C} 21-\mathrm{C} 22$ & -178.65 & -177.79 \\
\hline $\mathrm{C} 16-\mathrm{C} 18-\mathrm{C} 21-\mathrm{H} 43$ & 1.51 & 2.51 \\
\hline C20-C18-C21-C22 & 2.71 & 2.54 \\
\hline $\mathrm{C} 20-\mathrm{C} 18-\mathrm{C} 21-\mathrm{H} 43$ & -177.11 & -177.15 \\
\hline C17-S19-C20-C18 & -15.98 & -14.53 \\
\hline C17-S19-C20-C23 & 162.11 & 163.39 \\
\hline C18-C20-C23-C24 & 1.21 & 1.25 \\
\hline $\mathrm{C} 18-\mathrm{C} 20-\mathrm{C} 23-\mathrm{H} 45$ & 179.92 & 179.89 \\
\hline S19-C20-C23-C24 & -176.72 & -176.49 \\
\hline S19-C20-C23-H45 & 1.97 & 2.13 \\
\hline C18-C21-C22-C24 & -0.50 & -0.42 \\
\hline $\mathrm{C} 18-\mathrm{C} 21-\mathrm{C} 22-\mathrm{H} 44$ & 179.84 & 179.97 \\
\hline $\mathrm{H} 43-\mathrm{C} 21-\mathrm{C} 22-\mathrm{C} 24$ & 179.32 & 179.27 \\
\hline $\mathrm{H} 43-\mathrm{C} 21-\mathrm{C} 22-\mathrm{H} 44$ & -0.32 & -0.33 \\
\hline C2-C22-C24-C23 & -1.41 & -1.35 \\
\hline $\mathrm{C} 2-\mathrm{C} 22-\mathrm{C} 24-\mathrm{H} 46$ & -179.95 & -179.98 \\
\hline H44-C22-C24-C23 & 178.23 & 178.24 \\
\hline $\mathrm{H} 44-\mathrm{C} 22-\mathrm{C} 24-\mathrm{H} 46$ & -0.30 & -0.37 \\
\hline C20-C23-C24-C22 & 1.05 & 0.94 \\
\hline $\mathrm{C} 20-\mathrm{C} 23-\mathrm{C} 24-\mathrm{H} 46$ & 179.60 & 179.57 \\
\hline H45-C23-C24-C22 & -177.64 & -177.69 \\
\hline $\mathrm{H} 45-\mathrm{C} 23-\mathrm{C} 24-\mathrm{H} 46$ & 0.90 & 0.93 \\
\hline
\end{tabular}

\section{Vibrational Assignments}

A total of 52 atoms and 150 vibrational frequencies are observed in the spectroscopic study. The spectroscopic vibrational modes are calculated by HF and DFT/6-311+G (d, p) basis set. The frequencies, intensities of IR, Raman and characteristic group frequencies have also been calculated. In organic chemistry, vibrational spectroscopy is used to detect functional group ${ }^{13}$. It is also used to study molecular tests, kinematics, etc. The molecules, however are made up of different vibration modes, there is therefore a various vibrational spectrum in the study of the title molecule. Fig. 2 and 3 show the calculated frequencies. Table 2 shows the calculated and observed vibrational assignments with the HF and the B3LYP/6-311++G method.

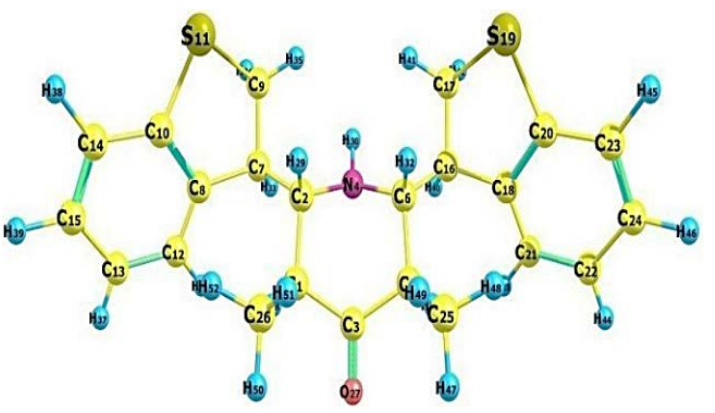

Fig. 1. Optimized structure of the organic molecule obtained at B3LYP/6-31G level of theory
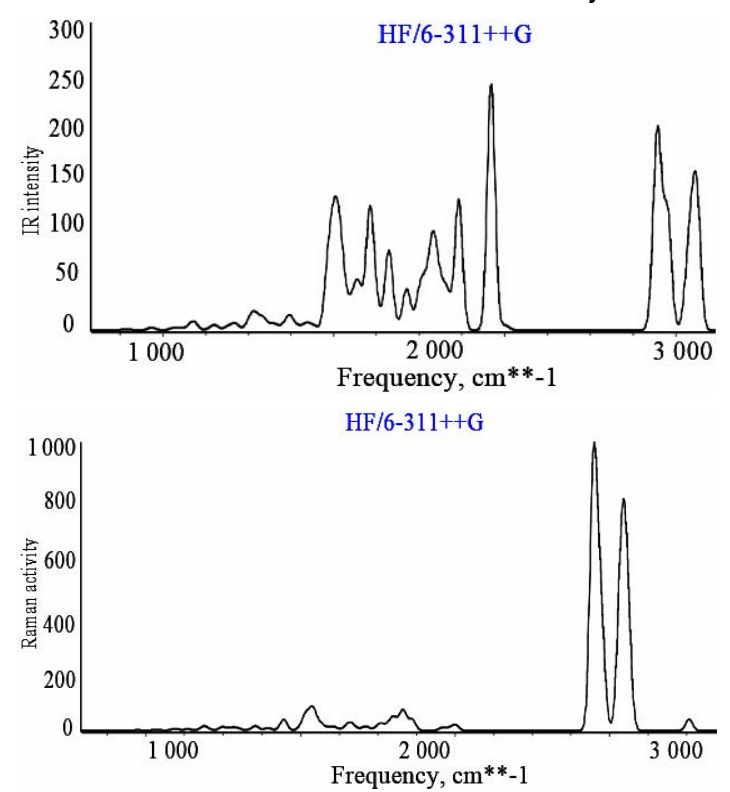

Fig. 2. Theoretical vibrational spectrum using HF/6-311++G for title molecule 

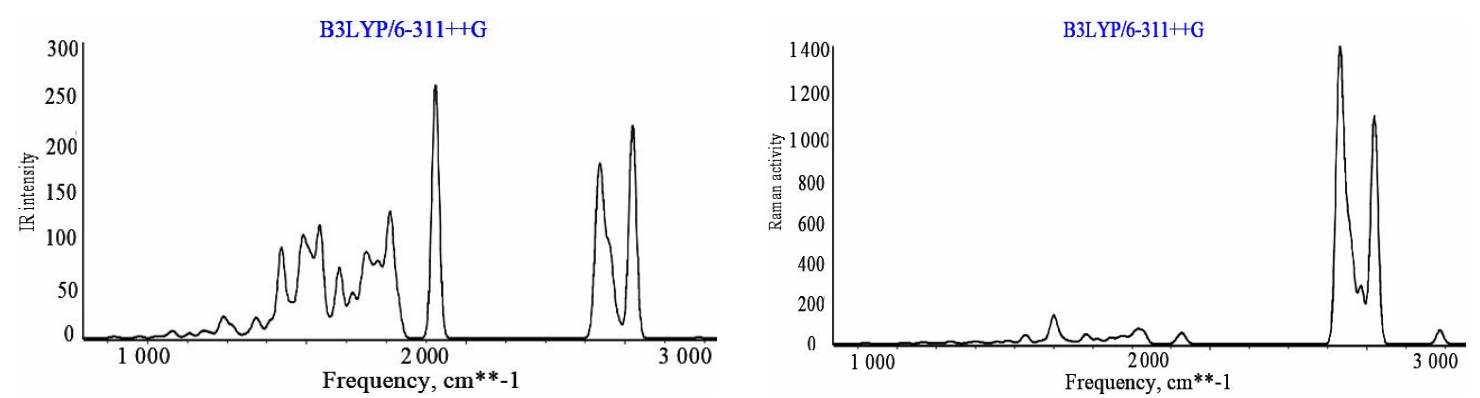

Fig. 3. Theoretical vibrational spectrum using B3LYP/6-311++G for title molecule

Table 2: Calculated vibrational frequencies $\left(\mathrm{cm}^{-1}\right)$ and assignments title compound based on HF/6-311++G $(d, p)$ and B3LYP/6311++G (d, p) methods

\begin{tabular}{|c|c|c|c|c|}
\hline \multicolumn{2}{|c|}{$\mathrm{HF}$} & \multicolumn{2}{|c|}{ B3LYP } & \multirow[t]{2}{*}{ Vibrational Assignments } \\
\hline $6-311 G$ & $6-311++G$ & $6-311 G$ & $6-311++G$ & \\
\hline-229.9653 & -229.9015 & -234.9466 & -219.1928 & Ring deformation \\
\hline-197.7089 & -197.4371 & -208.4349 & -190.6649 & $\begin{array}{l}\mathrm{C}-\mathrm{C} \text { wagging and ring } \\
\text { Deformation }\end{array}$ \\
\hline-64.792 & -66.0186 & -124.1784 & -51.1752 & Ring stretching \\
\hline-41.0665 & -41.8883 & -106.4637 & 17.6728 & Ring bending \\
\hline 58.4204 & 60.062 & -99.7258 & 54.9081 & $\begin{array}{l}\mathrm{C}=\mathrm{O} \text { wagging and ring } \\
\text { Twisting }\end{array}$ \\
\hline 75.373 & 75.0479 & 42.3406 & 63.7788 & $\begin{array}{l}\mathrm{C}=\mathrm{O} \text { wagging and ring } \\
\text { Twisting }\end{array}$ \\
\hline 99.3333 & 101.0364 & 66.7223 & 94.9203 & $\begin{array}{l}\mathrm{C}=\mathrm{O} \text { wagging and ring } \\
\text { Twisting }\end{array}$ \\
\hline 154.7052 & 153.7594 & 116.391 & 134.5907 & Ring breathing and twisting \\
\hline 178.8022 & 177.8274 & 141.2279 & 158.0064 & Ring bending \\
\hline 183.9821 & 183.2629 & 146.8225 & 159.746 & $\begin{array}{l}\text { Ring stretching and } \\
\text { C-H rocking }\end{array}$ \\
\hline 189.8117 & 188.5251 & 158.6888 & 165.8716 & Ring stretching \\
\hline 208.9179 & 209.0036 & 187.1049 & 190.4608 & Ring stretching \\
\hline 237.4691 & 237.5488 & 199.3726 & 205.6494 & $\begin{array}{l}\text { Ring deformation and } \\
\mathrm{C}-\mathrm{H} \text { rocking }\end{array}$ \\
\hline 266.7524 & 266.3947 & 228.7993 & 233.6933 & Ring stretching \\
\hline 272.5417 & 272.3757 & 238.9764 & 245.5082 & Ring breathing \\
\hline 288.6973 & 288.0281 & 248.7996 & 257.3432 & C-S out plan bending \\
\hline 306.8084 & 306.0891 & 269.2158 & 267.4396 & $\begin{array}{l}\text { Ring deformation and } \\
\mathrm{C}-\mathrm{H} \text { rocking }\end{array}$ \\
\hline 350.3618 & 350.9602 & 323.1495 & 319.4722 & Ring deformation \\
\hline 376.0731 & 377.3177 & 339.3296 & 335.6433 & $\mathrm{C}-\mathrm{H}$ rocking \\
\hline 389.7852 & 389.8144 & 343.792 & 341.0143 & Ring stretching \\
\hline 395.046 & 395.5424 & 360.9819 & 356.6177 & $\begin{array}{l}\mathrm{C}-\mathrm{H} \text { rocking and ring } \\
\text { Deformation }\end{array}$ \\
\hline 422.4164 & 423.1322 & 363.2598 & 360.3997 & $\begin{array}{l}\mathrm{C}-\mathrm{H} \text { rocking and ring } \\
\text { Deformation }\end{array}$ \\
\hline 429.9013 & 428.2786 & 390.2312 & 387.322 & Ring deformation \\
\hline 484.0331 & 482.8366 & 418.927 & 406.004 & $\mathrm{C}-\mathrm{H}$ rocking \\
\hline 493.7563 & 494.1814 & 459.9176 & 451.7972 & Ring deformation \\
\hline 514.0624 & 513.339 & 463.8629 & 458.6466 & $\begin{array}{l}\text { Ring deformation and } \\
\mathrm{C}-\mathrm{H} \text { rocking }\end{array}$ \\
\hline 519.1581 & 518.1794 & 466.8887 & 464.5253 & Ring deformation \\
\hline 538.5809 & 537.9589 & 490.1502 & 483.1624 & Ring deformation \\
\hline 550.413 & 549.7329 & 502.5644 & 494.4182 & Ring deformation \\
\hline 567.5258 & 567.3939 & 510.3295 & 501.7365 & $\begin{array}{l}\text { Ring deformation and } \\
\mathrm{C}-\mathrm{H} \text { wagging }\end{array}$ \\
\hline
\end{tabular}




\begin{tabular}{|c|c|c|c|c|}
\hline 582.7368 & 583.9799 & 531.9834 & 530.0417 & Ring stretching \\
\hline 600.9812 & 600.1037 & 539.9461 & 535.6637 & Ring breathing \\
\hline 616.14 & 616.7398 & 561.7379 & 553.4781 & Ring deformation \\
\hline 627.203 & 627.1937 & 575.4665 & 568.9549 & Ring deformation \\
\hline 646.0239 & 645.6668 & 589.3565 & 581.1875 & Ring deformation \\
\hline 655.1498 & 654.5796 & 603.8106 & 594.485 & $\begin{array}{l}\text { Ring deformation and } \\
\mathrm{N}-\mathrm{H} \text { rocking }\end{array}$ \\
\hline 698.1099 & 697.7662 & 642.6309 & 632.4736 & $\begin{array}{l}\text { Ring deformation and } \\
\mathrm{C}-\mathrm{H} \text { rocking }\end{array}$ \\
\hline 702.0815 & 701.5741 & 647.9494 & 635.5417 & $\begin{array}{l}\text { Ring deformation and } \\
\mathrm{C}-\mathrm{H} \text { rocking }\end{array}$ \\
\hline 714.0517 & 713.8855 & 649.8632 & 637.1369 & $\begin{array}{l}\text { Ring deformation and } \\
\mathrm{C}-\mathrm{H} \text { rocking }\end{array}$ \\
\hline 740.462 & 740.6503 & 687.0236 & 672.6354 & $\begin{array}{l}\text { Ring deformation and } \\
\mathrm{C}-\mathrm{H} \text { rocking }\end{array}$ \\
\hline 744.1232 & 744.0099 & 689.0506 & 677.9077 & $\begin{array}{l}\text { Ring deformation and } \\
\mathrm{C}=\mathrm{O}, \mathrm{N}-\mathrm{H} \text { wagging }\end{array}$ \\
\hline 785.8559 & 786.7782 & 727.7485 & 706.1245 & $\begin{array}{l}\text { Ring deformation and } \\
\mathrm{C}-\mathrm{H} \text { rocking }\end{array}$ \\
\hline 791.3242 & 791.3688 & 731.4054 & 713.5378 & $\begin{array}{l}\text { Ring deformation and } \\
\text { C-S stretching }\end{array}$ \\
\hline 839.1609 & 840.0747 & 753.0798 & 765.2063 & $\begin{array}{l}\mathrm{C}=\mathrm{O}, \mathrm{N}-\mathrm{H} \text { wagging and } \\
\text { ring deformation }\end{array}$ \\
\hline 870.9846 & 869.9239 & 791.343 & 774.9497 & $\begin{array}{l}\text { Ring deformation and } \\
\mathrm{C}-\mathrm{H} \text { wagging }\end{array}$ \\
\hline 881.1406 & 881.3927 & 792.3548 & 776.3088 & $\begin{array}{l}\text { Ring deformation and } \\
\mathrm{C}-\mathrm{H} \text { rocking }\end{array}$ \\
\hline 892.4164 & 892.1166 & 829.1346 & 816.7441 & $\begin{array}{l}\text { Ring breathing and } \\
\mathrm{C}-\mathrm{H} \text { rocking }\end{array}$ \\
\hline 914.724 & 914.705 & 841.0573 & 825.0769 & $\begin{array}{l}\text { Ring breathing and } \\
\mathrm{C}-\mathrm{H} \text { rocking }\end{array}$ \\
\hline 968.5293 & 966.2263 & 862.8071 & 839.9625 & $\mathrm{C}-\mathrm{H}$ wagging \\
\hline 996.142 & 994.4025 & 875.8364 & 847.6034 & $\mathrm{C}-\mathrm{H}$ wagging \\
\hline 1000.018 & 999.8391 & 891.3911 & 893.2602 & $\begin{array}{l}\text { Ring breathing and } \\
\mathrm{C}-\mathrm{H} \text { bending }\end{array}$ \\
\hline 1005.7455 & 1005.3395 & 908.6649 & 902.956 & $\begin{array}{l}\text { Ring deformation and } \\
\mathrm{C}-\mathrm{H} \text { wagging }\end{array}$ \\
\hline 1021.3447 & 1021.7178 & 949.602 & 925.617 & $\begin{array}{l}\text { C-H twisting and } \\
\text { C-S stretching }\end{array}$ \\
\hline 1032.097 & 1033.9909 & 964.1402 & 935.1974 & C-S stretching \\
\hline 1034.5141 & 1036.325 & 977.5419 & 950.2878 & $\mathrm{C}-\mathrm{H}$ wagging \\
\hline 1044.7215 & 1045.3036 & 987.4671 & 958.3716 & $\mathrm{C}-\mathrm{H}$ wagging \\
\hline 1057.2137 & 1057.681 & 989.5713 & 968.7278 & $\mathrm{C}-\mathrm{H}$ twisting \\
\hline 1078.7418 & 1079.0423 & 998.8103 & 982.6365 & $\begin{array}{l}\text { Ring deformation and } \\
\mathrm{C}-\mathrm{H} \text { rocking }\end{array}$ \\
\hline 1086.7718 & 1087.4327 & 1003.6359 & 986.2575 & $\begin{array}{l}\text { Ring breathing and } \\
\mathrm{C}-\mathrm{H} \text { twisting }\end{array}$ \\
\hline 1095.0551 & 1095.8576 & 1013.132 & 1000.3396 & Ring breathing \\
\hline 1104.1023 & 1103.1149 & 1033.6935 & 1011.2327 & $\begin{array}{l}\text { Ring deformation and } \\
\mathrm{C}-\mathrm{H} \text { twisting }\end{array}$ \\
\hline 1116.1952 & 1115.5555 & 1040.6599 & 1015.7436 & $\begin{array}{l}\text { Ring deformation and } \\
\mathrm{C}-\mathrm{H} \text { rocking }\end{array}$ \\
\hline 1123.0419 & 1123.4044 & 1046.1253 & 1017.5342 & $\begin{array}{l}\text { Ring deformation and } \\
\mathrm{C}-\mathrm{H} \text { rocking }\end{array}$ \\
\hline 1128.275 & 1129.2571 & 1052.0511 & 1022.2435 & $\begin{array}{l}\text { Ring deformation and } \\
\mathrm{C}-\mathrm{H} \text { rocking }\end{array}$ \\
\hline 1132.8151 & 1133.3653 & 1053.9672 & 1027.8967 & $\begin{array}{l}\text { Ring deformation and } \\
\mathrm{C}-\mathrm{H} \text { rocking }\end{array}$ \\
\hline 1136.4018 & 1137.4183 & 1061.0005 & 1031.9701 & $\begin{array}{l}\text { Ring deformation and } \\
\mathrm{C}-\mathrm{H} \text { rocking }\end{array}$ \\
\hline
\end{tabular}




\begin{tabular}{|c|c|c|c|c|}
\hline 1138.8026 & 1138.944 & 1061.7903 & 1038.7215 & $\begin{array}{l}\text { Ring deformation and } \\
\mathrm{C}-\mathrm{H} \text { rocking }\end{array}$ \\
\hline 1149.4985 & 1149.7295 & 1076.3219 & 1052.9522 & $\begin{array}{l}\text { Ring deformation and } \\
\mathrm{C}-\mathrm{H} \text { rocking }\end{array}$ \\
\hline 1174.7591 & 1174.0216 & 1079.659 & 1058.2829 & $\begin{array}{l}\text { Ring deformation and } \\
\mathrm{C}-\mathrm{H} \text { twisting }\end{array}$ \\
\hline 1177.4643 & 1177.3652 & 1087.926 & 1065.6865 & $\begin{array}{l}\text { Ring deformation and } \\
\mathrm{C}-\mathrm{H} \text { rocking }\end{array}$ \\
\hline 1186.2782 & 1186.3641 & 1100.8396 & 1083.9865 & $\begin{array}{l}\text { Ring deformation and } \\
\mathrm{C}-\mathrm{H} \text { twisting }\end{array}$ \\
\hline 1192.9383 & 1193.1815 & 1103.1899 & 1085.0626 & $\begin{array}{l}\text { Ring deformation } \\
\mathrm{C}-\mathrm{H} \text { rocking }\end{array}$ \\
\hline 1193.7124 & 1194.7236 & 1130.4886 & 1109.2998 & $\begin{array}{l}\text { Ring deformation and } \\
\mathrm{C}-\mathrm{H} \text { wagging and rocking }\end{array}$ \\
\hline 1212.3907 & 1210.1853 & 1143.4984 & 1122.4819 & $\begin{array}{l}\text { Ring deformation and } \\
\mathrm{C}-\mathrm{H} \text { rocking }\end{array}$ \\
\hline 1213.6868 & 1211.2829 & 1150.3136 & 1128.6193 & $\begin{array}{l}\text { Ring deformation and } \\
\mathrm{C}-\mathrm{H} \text { twisting }\end{array}$ \\
\hline 1226.1481 & 1224.5593 & 1165.8715 & 1139.0741 & $\begin{array}{l}\text { Ring deformation and } \\
\mathrm{C}-\mathrm{H} \text { rocking }\end{array}$ \\
\hline 1235.7552 & 1234.6732 & 1172.9482 & 1142.1047 & $\mathrm{C}-\mathrm{H}$ bending \\
\hline 1260.5388 & 1258.0619 & 1178.5707 & 1149.614 & $\begin{array}{l}\text { Ring deformation and } \\
\mathrm{C}-\mathrm{H} \text { rocking }\end{array}$ \\
\hline 1279.8835 & 1277.546 & 1185.4593 & 1167.0785 & $\begin{array}{l}\text { Ring deformation and } \\
\mathrm{C}-\mathrm{H} \text { wagging }\end{array}$ \\
\hline 1305.1793 & 1304.1336 & 1195.6647 & 1181.6884 & $\begin{array}{l}\text { Ring deformation and } \\
\mathrm{C}-\mathrm{H} \text { twisting }\end{array}$ \\
\hline 1310.901 & 1309.6981 & 1223.7745 & 1198.9568 & $\begin{array}{l}\text { Ring deformation and } \\
\mathrm{C}-\mathrm{H} \text { rocking }\end{array}$ \\
\hline 1323.6257 & 1320.8807 & 1230.3274 & 1205.3265 & $\begin{array}{l}\text { Ring deformation and } \\
\mathrm{C}-\mathrm{H} \text { rocking }\end{array}$ \\
\hline 1334.0922 & 1331.7754 & 1238.3741 & 1205.8497 & $\begin{array}{l}\text { Ring deformation and } \\
\mathrm{C}-\mathrm{H} \text { rocking }\end{array}$ \\
\hline 1338.3194 & 1335.5194 & 1251.4193 & 1227.2436 & $\begin{array}{l}\text { Ring deformation and } \\
\mathrm{C}-\mathrm{H} \text { rocking }\end{array}$ \\
\hline 1342.9036 & 1340.1095 & 1255.4694 & 1228.631 & $\mathrm{C}-\mathrm{H}$ twisting and wagging \\
\hline 1348.5897 & 1346.8178 & 1266.4931 & 1238.9583 & $\mathrm{C}-\mathrm{H}$ twisting and rocking \\
\hline 1381.9072 & 1378.3352 & 1272.0954 & 1242.1408 & $\mathrm{C}-\mathrm{H}$ twisting and rocking \\
\hline 1400.2423 & 1395.7662 & 1281.4487 & 1245.7012 & $\mathrm{C}-\mathrm{H}$ twisting and rocking \\
\hline 1415.1201 & 1412.235 & 1285.594 & 1262.0967 & $\mathrm{C}-\mathrm{H}$ twisting \\
\hline 1420.8765 & 1417.899 & 1300.6325 & 1268.0356 & $\mathrm{C}-\mathrm{H}$ wagging \\
\hline 1425.1358 & 1421.0844 & 1307.8837 & 1277.7265 & $\mathrm{C}-\mathrm{H}$ wagging \\
\hline 1431.8808 & 1429.0995 & 1310.5914 & 1290.8372 & $\mathrm{C}-\mathrm{H}$ twisting \\
\hline 1436.6867 & 1434.0305 & 1325.1579 & 1296.0829 & $\mathrm{C}-\mathrm{H}$ twisting \\
\hline 1447.8105 & 1445.6143 & 1336.6448 & 1308.1699 & $\mathrm{C}-\mathrm{H}$ rocking \\
\hline 1494.91 & 1492.5972 & 1368.7083 & 1337.9642 & $\mathrm{C}-\mathrm{H}$ wagging \\
\hline 1512.7839 & 1509.2183 & 1378.6874 & 1351.1258 & $\mathrm{C}-\mathrm{H}$ wagging \\
\hline 1517.5159 & 1514.8413 & 1389.4823 & 1357.1497 & $\mathrm{C}-\mathrm{H}$ wagging \\
\hline 1523.0336 & 1519.6343 & 1394.0807 & 1366.8168 & $\mathrm{C}-\mathrm{H}$ twisting \\
\hline 1531.587 & 1528.0701 & 1403.0751 & 1369.4993 & C-H Wagging \\
\hline 1549.5016 & 1541.9211 & 1411.9181 & 1379.2735 & C-H Wagging \\
\hline 1567.0541 & 1560.6581 & 1430.6842 & 1390.1801 & $\mathrm{C}-\mathrm{H}$ Wagging \\
\hline 1567.5519 & 1562.0163 & 1433.5468 & 1393.8097 & C-H Wagging \\
\hline 1570.3472 & 1567.9337 & 1435.7215 & 1409.3893 & $\mathrm{C}-\mathrm{H}$ rocking \\
\hline 1581.7624 & 1577.566 & 1446.5708 & 1417.6663 & C-H Wagging \\
\hline 1586.0539 & 1582.3054 & 1456.4304 & 1427.632 & C-H Wagging \\
\hline 1587.7337 & 1584.0311 & 1459.948 & 1438.6632 & $\mathrm{C}-\mathrm{H}$ rocking \\
\hline 1595.4597 & 1592.1041 & 1473.682 & 1444.8595 & C-H rocking \\
\hline 1603.1257 & 1599.8848 & 1475.1923 & 1446.3295 & C-H Wagging \\
\hline 1610.5187 & 1607.148 & 1482.1595 & 1449.7688 & $\mathrm{C}-\mathrm{H}$ rocking \\
\hline
\end{tabular}


S, R. K., Orient. J. Chem., Vol. 37(6), 1396-1408 (2021)

\begin{tabular}{|c|c|c|c|c|}
\hline 1613.0798 & 1608.8684 & 1484.7331 & 1451.7971 & C-H Wagging \\
\hline 1613.8459 & 1609.7811 & 1496.3276 & 1467.7979 & C-H Wagging \\
\hline 1626.5013 & 1621.3002 & 1499.5117 & 1471.5591 & $\mathrm{C}-\mathrm{H}$ rocking \\
\hline 1642.0802 & 1637.7192 & 1511.4255 & 1482.1718 & $\mathrm{C}-\mathrm{H}$ rocking \\
\hline 1655.5896 & 1651.8691 & 1531.0755 & 1497.2362 & $\mathrm{C}-\mathrm{H}$ bending \\
\hline 1657.8567 & 1655.6736 & 1532.3556 & 1501.4131 & $\mathrm{C}-\mathrm{H}$ bending \\
\hline 1661.2425 & 1658.9591 & 1535.5841 & 1504.857 & $\mathrm{C}-\mathrm{H}$ bending \\
\hline 1664.4556 & 1660.9151 & 1547.6845 & 1523.0464 & $\mathrm{~N}-\mathrm{H}$ bending \\
\hline 1668.8901 & 1665.5444 & 1552.7418 & 1524.1664 & $\mathrm{C}-\mathrm{H}$ bending \\
\hline 1678.7874 & 1675.2564 & 1557.3609 & 1535.1664 & $\mathrm{C}-\mathrm{H}$ rocking \\
\hline 1722.0965 & 1720.0198 & 1563.1688 & 1537.6116 & Ring deformation and $\mathrm{C}-\mathrm{H}$ \\
\hline 1727.1414 & 1725.064 & 1592.3086 & 1571.1272 & Bending Ring vibration \\
\hline 1734.8125 & 1731.6091 & 1597.9645 & 1575.8544 & $\mathrm{C}-\mathrm{H}$ bending Ring vibration \\
\hline 1928.6092 & 1920.2172 & 1810.5681 & 1792.4077 & $\mathrm{C}-\mathrm{H}$ bending \\
\hline \multicolumn{5}{|c|}{$\mathrm{C}-\mathrm{H}$ Wagging } \\
\hline 1975.8466 & 1974.1168 & 1839.9844 & 1809.6942 & Ring deformation \\
\hline 2007.1174 & 2005.5411 & 1858.1994 & 1828.6638 & $\mathrm{C}-\mathrm{H}$ Wagging \\
\hline \multicolumn{5}{|c|}{ C-H Wagging } \\
\hline 2868.4597 & 2870.0092 & 2795.3312 & 2816.6257 & C-H symmetric Stretching \\
\hline 2879.9917 & 2879.9549 & 2801.5365 & 2822.418 & C-H Symmetric Stretching \\
\hline 2881.5956 & 2883.5014 & 2804.7972 & 2831.4775 & C-H Asymmetric Stretching \\
\hline 2891.1491 & 2892.8767 & 2810.7297 & 2835.1639 & C-H Symmetric Stretching \\
\hline 2893.1229 & 2893.476 & 2823.5487 & 2848.9838 & C-H Symmetric Stretching \\
\hline 2895.05 & 2896.9 & 2826.5636 & 2851.9813 & C-H Symmetric Stretching \\
\hline 2912.5909 & 2914.9605 & 2852.112 & 2875.7023 & C-H Asymmetric Stretching \\
\hline 2913.9058 & 2916.2647 & 2853.5064 & 2876.6501 & $\mathrm{C}-\mathrm{H}$ Symmetric Stretching \\
\hline 2919.5906 & 2921.949 & 2858.3856 & 2881.0983 & C-H Asymmetric Stretching \\
\hline 2921.0254 & 2923.5672 & 2859.0394 & 2882.1881 & C-H Asymmetric Stretching \\
\hline 2934.2185 & 2934.3405 & 2865.9662 & 2884.1242 & C-H Asymmetric Stretching \\
\hline 2946.8574 & 2948.1615 & 2883.7547 & 2902.3975 & $\mathrm{C}-\mathrm{H}$ symmetric Stretching \\
\hline 2947.8394 & 2949.3087 & 2885.4393 & 2904.5105 & C-H Asymmetric Stretching \\
\hline 2953.0494 & 2954.3067 & 2886.3938 & 2905.7117 & C-H Stretching \\
\hline 2958.2998 & 2959.8465 & 2903.499 & 2920.3287 & C-H Asymmetric Stretching \\
\hline 3059.1811 & 3059.7431 & 2947.2746 & 2962.7994 & $\mathrm{C}-\mathrm{H}$ Asymmetric Stretching \\
\hline 3069.196 & 3065.3724 & 3005.9766 & 3027.1185 & C-H Asymmetric Stretching \\
\hline 3069.4009 & 3065.6188 & 3006.2641 & 3027.4497 & C-H Asymmetric Stretching \\
\hline 3082.6205 & 3079.2146 & 3017.6493 & 3036.9308 & C-H Asymmetric Stretching \\
\hline 3082.864 & 3079.4807 & 3017.9073 & 3037.2391 & $\mathrm{C}-\mathrm{H}$ Asymmetric Stretching \\
\hline 3100.1909 & 3096.564 & 3031.4313 & 3047.9356 & C-H Asymmetric Stretching \\
\hline 3100.3236 & 3096.7068 & 3032.8139 & 3049.4982 & $\mathrm{C}-\mathrm{H}$ Asymmetric Stretching \\
\hline 3125.0966 & 3123.3264 & 3035.8762 & 3050.0402 & C-H Stretching \\
\hline 3127.4855 & 3125.7954 & 3037.5426 & 3052.6164 & $\mathrm{C}-\mathrm{H}$ Stretching \\
\hline 3509.2952 & 3514.3522 & 3448.0997 & 3464.4121 & $\mathrm{~N}-\mathrm{H}$ Stretching \\
\hline
\end{tabular}

\section{C-H vibrations}

A C-H stretching vibration in the region $3,100-3000 \mathrm{~cm}^{-1}{ }^{14}$ has been shown by the hetero aromatic structure. This is the only area where the $\mathrm{C}-\mathrm{H}$ stretching vibration can be identified. The nature of the substitutions in this region does not greatly affect the bands. Due to the influence from N-H stretching vibration, which was found very dominant in this molecule. The vibrational frequency in the region of 2816-1142 $\mathrm{cm}^{-1}$ was evident of title molecule in this study. In the expected range of vibration, the functional group vibrations were observed.

\section{$\mathrm{N}-\mathrm{H}$ vibration}

The $\mathrm{N}-\mathrm{H}$ stretching frequencies of vibration

are always higher than the other stretching vibrations, and the length of the bond is smaller than the length of other molecules. Usually $\mathrm{N}-\mathrm{H}$ stretching vibrations ${ }^{15}$ are continued at $3500-3300 \mathrm{~cm}^{-1}$ for any heterocyclic compound. Six vibrational modes are required by nitrogen hydrogen bonds and this is part of the entire vibrational pattern. The $\mathrm{N}-\mathrm{H}$ vibration takes place in the area $3464 \mathrm{~cm}^{-1}$ in this research. This is due to strong hydrogen bonding during the gas phase, whereas in the solid phase it is absent.

\section{$\mathrm{C}=\mathbf{O}$ vibrations}

The $\mathrm{C}=\mathrm{O}$ stretching vibration band visualized from the carboxylic group in which the $\mathrm{C}=\mathrm{O}$ stretching position is dependent on physical 
state, mass and electronic effects, intermolecular and intra molecular hydrogen bonding ${ }^{16}$, usually in this region between 1750 and $1700 \mathrm{~cm}^{-1}$. This band position determines the bond strength of the hydrogen. In this research the carboxyl group, assigned below $1000 \mathrm{~cm}^{-1}$ in the region, are directly linked to its electronic structure as well as its geometric positioning. The effect of the inductive, mesomeric and conjugating efficiency in both inter and intermolecular factors on carbonyl absorption of frequent organic compounds.

\section{C-C Vibrations}

The visible spectrum of the benzene and its derivatives, the ring vibration is very remarkable and characteristic of the aromatic ring itself. This is not so much because of the nature of the substituent but because of the way the chain is substituted. In this study, the title molecule found that the region is generally observed below $700 \mathrm{~cm}^{-1}$ at external vibration bending. The chains vibrations were observed at $190 \mathrm{~cm}^{-1}$.

\section{Ring vibrations}

The benzene ring has six stretching vibration. The bending vibration of the benzene ring in the plane and out of the plane is usually seen in the literature below $1000 \mathrm{~cm}^{-1}$, and those modes are non-pure but are significantly affected by other vibrations. The title molecule affects to a great extent the in-plan and out-of-plane bending modes of the rings, producing bands less than $600 \mathrm{~cm}^{-1}$.

\section{C-S vibrations}

The C-S stretching bands are normally noticed in the range of $670-930 \mathrm{~cm}^{-1}$. For our study, the title compound shows the frequency range from 713 to $935 \mathrm{~cm}^{-1}$. The out-of-plane C-S bending vibrations bands are predicted in the regions $420-320 \mathrm{~cm}^{-1}$, respectively. In the current research work, the $\mathrm{C}-\mathrm{S}$ out of plane bending vibrations are assigned to below $300 \mathrm{~cm}^{-1}$. All the $\mathrm{C}-\mathrm{S}$ vibrational bands of the molecule are well determined with the previous research work. Also, the observed values by $B 3 L Y P / 6-311++G(d, p)$ are almost related to the computed values.

\section{Mulliken atomic charge}

The effect of atomic charges determines the molecular polarization, dipole moment, electronic structure and few molecular system properties.
The classification of charges across the atoms proposes that donor and recipient pair molecules are established. The investigation of Mulliken atomic charges plays a vital role in the implementation of quantum chemicals in the molecular system. Fig. 4 shows the Mulliken charge arrangement structure of the title compound. The negative charge carrier is more in oxygen and Sulphur atoms, while positive charge occupies all hydrogen atoms. In addition, the S19 atom has a greater electron-negative $(-0.38886 e)$ charge than the S11 atom in Mulliken atomic chargers. This is because the electronnegative Sulphur atom is present. Thus Mulliken population analysis interprets the reaction behavioral analysis of various chemicals.

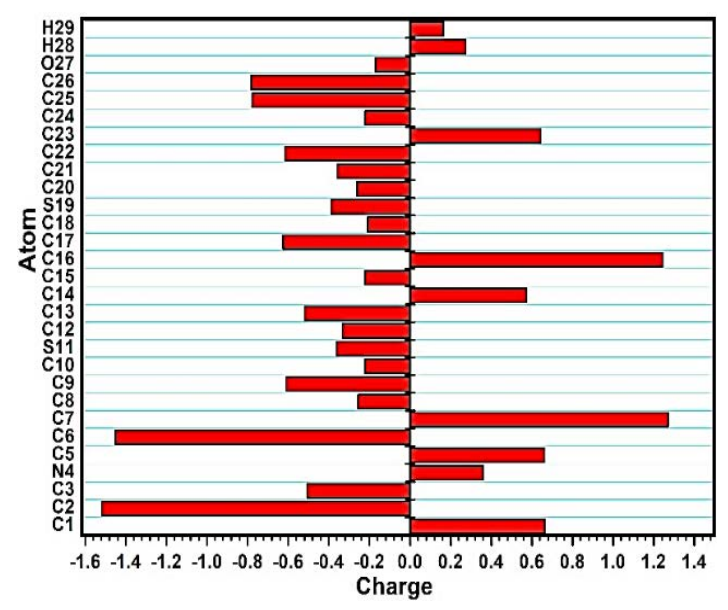

Fig. 4. Schematic representation of Mullikan atomic charges Frontier molecular orbitals

The frontier molecular orbitals play an important role in understanding the electrical and optical properties of the titled molecule. The HOMO energy defines the ability to supply electrons while the LUMO shows that electrons can be accepted. The molecular chemical ability is defined by the HOMOLUMO gap. A molecule with a small energy gap in the orbit is more polarized and usually combined with the high chemical responsiveness. The energy gap between HOMO and LUMO is more influential for structural stability. Fig. 5 shows the difference in energy level between HOMO and LUMO. The estimated energy difference is $9.3387 \mathrm{eV}$. This wide energy gap therefore means high excitement, good stability and high chemical durability.

\footnotetext{
HOMO energy $=-8.4165 e \mathrm{~V}$

LUMO energy $=0.9222 \mathrm{eV}$ HOMO-LUMO energy gap $=9.3387 \mathrm{eV}$
} 
The smaller energy band gap increases the chemical activity of the titled molecule. The HOMO-LUMO energy and its gap are determined using $\mathrm{HF} / 6-11++\mathrm{G}(\mathrm{d}, \mathrm{p})$ level.

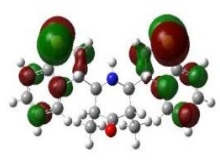

Еномо $=-8.4165 \mathrm{eV}$

HOMO

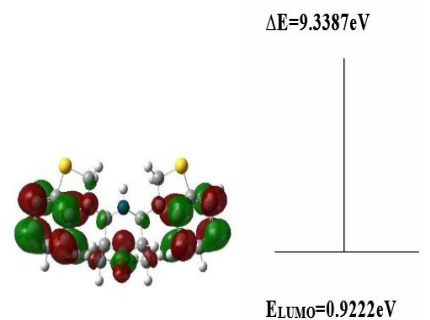

LUMO

Fig. 5. The frontier molecular orbitals of title molecule

\section{Molecular orbital studies}

Furthermore, the difference of energy band values is known to help us identify the chemical reactivity and stability of titled molecule. The energy band gap of the molecule is small and this gap is usually combined with a high reactivity of chemicals and a low kinetic stability. In terms of the degree of orbital reactivity of the atom ${ }^{17}$ the quantum chemical calculations are performed. Mulliken was introduced a new formulation, which allowed to extend the concept to molecules, in terms of two further periodic properties, namely ionizing potentials and affinity. The affinity of electron refers exactly to the ability to accept an electron. The greater
HOMO-LUMO gap means a hard molecule, and a smaller HOMO-LUMO gap means a soft molecule, due to its chemical hardness. The reactivity of the selected compound is also associated with the molecule's hardness. The values of ionization and electron affinity are calculated with both HOMO and LUMO. For Mulliken electron negativity, the mean of HOMO and LUMO energy value can be used. The index of electrophility is the amount of energy reduction due to the greater electron movement between donor and recipient. The values of electronegativity, chemical potential, chemical hardness, softness, and electrophility index are $3.74715 \mathrm{eV},-3.74715 \mathrm{eV}, 4.66935 \mathrm{eV}, 0.107081 \mathrm{eV}$, $1.503543 \mathrm{eV}$, respectively, for the title Compound.

\section{Thermodynamic Parameters}

Several thermodynamic parameters such as heat capacity, entropy and enthalpy changes for the titled compound were determined using, the DFT/B3LYP with 6-31G (d, p) basic sets. Such estimated frequencies of the title compound provide the information of all structural thermodynamic characteristics including rotational constants, null point vibration power, heat capacity, and entropy ${ }^{18}$. These values are calculated using the basis set of $6-311++G(d, p)$ levels using B3LYP and HF method and presented in the Table 3. For further understanding the selected molecules, the collected thermodynamic parameters are used to analysis the thermodynamic functional relationships and the direction of chemical reactions in accordance with the thermodynamics law.

Table 3: The calculated thermodynamic parameter of title molecule

\begin{tabular}{|c|c|c|c|c|}
\hline Basis set & HF 6-311G & $\mathrm{HF} 6-311++\mathrm{G}$ & B3LYP 6-311G & B3LYP $6-311++G$ \\
\hline Zero-Point Vibrational & 290.6913 & 290.4561 & 273.5251 & 271.3272 \\
\hline Energy(Kcal mol-1) & 0.34304 & 0.34304 & 0.34304 & 0.34304 \\
\hline Rotational Constants & 0.09194 & 0.09194 & 0.09194 & 0.09194 \\
\hline Frequency $(\mathrm{GHz})$ & 0.07810 & 0.07810 & 0.07810 & 0.07810 \\
\hline Specific heat(Cv) (Cal mol-1K-1) & 72.466 & 72.518 & 78.239 & 83.756 \\
\hline Entropy(S) (Cal mol-1K-1) & 128.996 & 128.979 & 133.915 & 143.786 \\
\hline Dipole Moment $\mu$ (Debye) & 4.7568 & 4.6720 & 4.0293 & 3.6527 \\
\hline
\end{tabular}

\section{Molecular electrostatic potential (MEP) mapping}

The electrostatic potential diagram illustrates the probability that the electron densities are integrated throughout the system. In other words, it provides functional information to the reactive section. Electrophilic and nucleophilic molecular system centers are suitable for the development of biochemical system. The electrostatic potential of the title compound shown in Fig. 6. The molecular surface contours are also illustrated in Fig. 7. The region with the most negative potential is shown in a red colour in the MEP diagram, while the blue colour shows the most positive potential surface. Red, blue, light blue, yellow and green are shown on the surface of the MEP. 
Red surface is electron-rich and moderately negative; blue, electron-deficient and partially positive; light-blue, light electron-deficient; yellow colour; a type of region rich in electrons; and green, neutral.

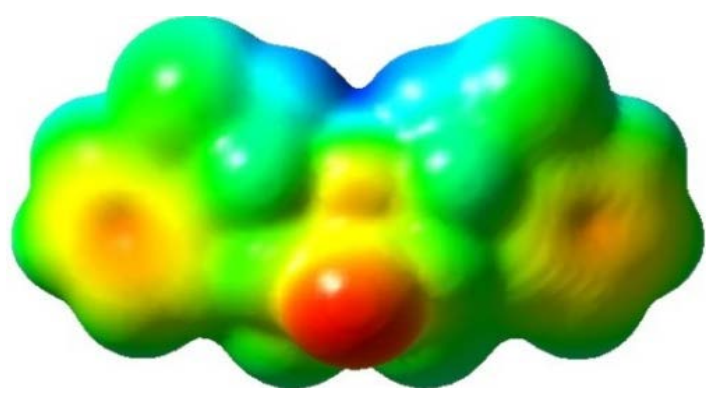

Fig. 6. Molecular electrostatic potential surface

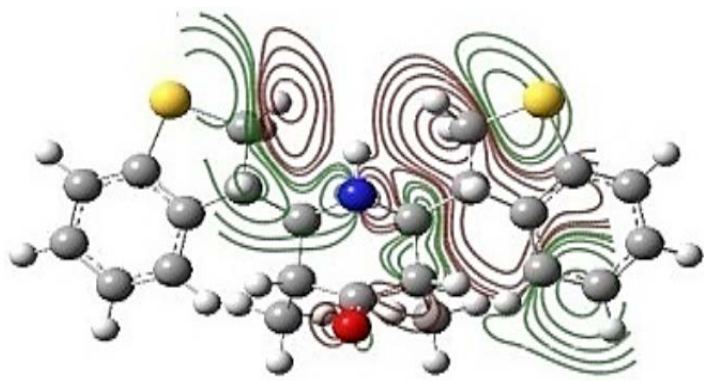

Fig. 7. MEP contour of title molecule

\section{Hyperpolarizability studies}

As an extensive tool for research on spectroscopy analysis, hyper polarizability and the non-linear optical properties of the titled molecule of potential NLO materials are considered. Nonlinear response, Hyperpolarizability ( $\beta \mathrm{ijk}$ ) and linear polarization (aij) of first order may be furnished as Taylor expansion in the form $\mu_{\text {tot }}=\mu_{0}+a_{i j} E_{i}+\beta_{i j k} E_{i} E_{j}+\ldots$

The dipole moment $(\mu)$, mean polarizability $(\alpha)$ and the first order Hyperpolarizability $(\beta)^{19}$ for the titled molecule is illustrated using following equations:

$$
\begin{gathered}
\mu=\sqrt{\left(\mu_{x}{ }^{2}+\mu_{x}{ }^{2}+\mu_{x}{ }^{2}\right)} \\
\alpha=\frac{\left(\alpha_{x x}+\alpha_{y y}+\alpha_{z z}\right)}{3}
\end{gathered}
$$

$\beta=\left[\left(\beta_{x x x}+\beta_{x y y}+\beta_{x z z}\right)^{2}+\left(\beta_{y y y}+\beta_{y z z}+\beta_{y x x}\right)^{2}+\left(\beta_{z z z}+\beta_{z x x}\right.\right.$ $\left.\left.+\beta_{z y y}\right)^{2}\right]^{(1 / 2)}$

The estimated dipole moment, mean polarizability and first order hyperpolarization of first-order values are 7,478 Debye, 0,402 $\AA$ esu and $1,4024 \times 10^{-31} \mathrm{~cm}^{5} \mathrm{esu}^{-1}$. A $\beta$-electron donor-to- acceptor movement which makes the molecule highly polarizable as a vital behavior for NLO activities ${ }^{20}$.

\section{CONCLUSION}

The results presented and discussed in the report of procedure seemed appropriate to be summarized. The theoretical analysis of DFT provides information on the orbital interactions and vibrational frequency systems, nature of the electronic structure, functional groups. In the organic synthesis of perfume, drugs, dyes and pharmaceuticals, heterocyclic compounds play significant role. The use of such a base set of 6-311 and $6-311++$ is availed to optimize the geometry of piperidine products by HF and DFT/B3LYP methods. The computed data is based on proof of the gaseous phase. The different calculated bonding angles are satisfactorily compatible with the normal values. The B3LYP/6- $311++G(d$, $p)$, then used to generate dependable geometry and associated piperidine derivatives properties. The method further tends to produce a vibration analysis. The orbital bond analysis provides the natural structure of Lewis with maximum accuracy. The highest rate of electron density will be achieved. In addition, the HOMO-LUMO band gap assists the molecule's biologically active properties. It provides information on the distribution of the charge density and differentiates the chemical molecule reactivity. The mapping electron density with electrostatic potential surface has been achieved to explain the size, the shape, the charging density distribution and the location of the chemical reactivity of the molecules. Finally, the Hyperpolarizability of the tile compound is analyzed for optical industrial applications.

\section{ACKNOWLEDGMENT}

We are extremely indebted to Dr. Sudha Mohan ram, Principal of Sri Eshwar College of Engineering, Coimbatore for the steadfast encouragement during all stages of our research to realize our work into reality.

\section{Conflict of Interest}

The authors claim no conflict of interest in the current research work. 


\section{REFERENCES}

1. Subbagh H.I. El, AbuZaid S.M., Mahran M.A., Badira F.A, Alobaid A.M., J. Med. Chem., 2000, 43, 2915-2921.

2. Jeron B.R., Spencer K.H., Eur. Pat. Appl. Ep., 1988, 27, 7794.

3. Perumal R.V., Adiraj M., Shanmugapandiyan P., Indian Drugs., 2001, 38, 156-159.

4. Hagenbach R.E., Gysin H., Experiential., 1952, 8, 184-185.

5. Katritzky A.R., Fan W. J, J. Org. Chem., 1990, 55, 3205-3209.

6. Ganelin C.R., Spickett R.G, J. Med. Chem.,1965, 8, 619-625.

7. Savithiri.S, Arockia doss.M, Rajarajan. G, Thanikachalam.V, Bharanidharan.S, Saleem. $\mathrm{H}$, Spectrochim. Acta Part A., 2015, 136, 782-792.

8. Arockia doss.M, Savithiri.S, Rajarajan.G, Thanikachalam.V, Saleem.H, Spectrochim. Acta Part A., 2015, 148, 189-202.

9. Arockia doss.M, Savithiri.S, Rajarajan.G, Thanikachalam.V, Saleem.H, Spectrochim. Acta Part A., 2015, 151, 773-784.

10. Anandhy.K, Arockia doss.M, Savithiri.S, Rajarajan.G, Mahalakshmi.S, Int. J. Adv. Res. Trends Eng. Technol., 2016, 3(2), 1301-1311.
11. Stratmann.R. E, Scuseria G.E, Frisch. M. J, J. Chem. Phys., 1998, 109, 8218-8224.

12. Miertus. S, Tomasi. J, Chem. Phys., 1982, 65, 239-245.

13. Snehalatha.M, Ravi Kumar. Joe C, I.H., Sekar.N, Jayakumar V.S, Spectrochim. Acta Part A., 2009, 72, 654-662.

14. Varsanyi.G, Vibrational Spectra of Benzene Derivatives, Academic Press, Newyork., 1980.

15. Chandra. S, Saleem. H, Sudaraganesan. N, Sebastian. S, Spectrochim Acta., 2008, A74 704-713.

16. Mohanbabu. B, Bharathikannan. R, Siva. G, J Mater Sci: Mater Electron., 2017, 28, 13740-13749.

17. Nabil Elleuch, Walid Amamou, Ali Ben Ahmed, Younes Abid, Habib Feki, J. Spect. Chem. Acta Part A., 2014, 128, 781.

18. Govindarajan M., Karabacak M., Udayakumar V., Periandy S., "Spectrochimica Acta PartA., 2012, 88, 37.

19. Zhang R, Dub B, Sun G, Sun Y, Spectrochim. Acta A., 2010, 75, 1115.

20. Ramalingam S, Periandy S, Mohan S, Spectrochimica Acta Part., 2010, A 77, 73. 